\title{
Fachbeitrag
}

\section{Andreas Ledl \\ Software, Server, Suchmaschine - Technische Kriterien der Gründung und des Betriebs von (Diamond) Open Access-Zeitschriften}

DOI 10.1515/abitech-2017-0005

Zusammenfassung: Der vorliegende Beitrag beschreibt, wie mit Hilfe non-proprietärer Software eine technische Publikationsinfrastruktur für unabhängige Open-AccessZeitschriften aufgesetzt werden kann. Er stellt eine Auswahl an Systemen zur Verwaltung und Veröffentlichung wissenschaftlicher Journals sowie Tools zur Erzeugung verschiedener Artikel-Formate vor. Darüber hinaus werden Möglichkeiten des Hostings und der Indexierung einer Zeitschrift thematisiert. Schließlich präsentiert er mit PubPub eine benutzerfreundliche und für Anhänger von mehr Offenheit im wissenschaftlichen Publikationswesen zukunftsträchtige Alternative zu OJS.

Schlüsselwörter: Open-Access-Zeitschrift, Publikationsinfrastruktur, Publikationswerkzeuge

\section{Software, server, search engine - technical criteria of establishing and running (Diamond) Open Access journals}

Abstract: This article describes how non-proprietary software can be used to create a technical publishing infrastructure for independent Open Access journals. It introduces a selection of systems for the administration and publication of academic journals as well as tools for the creation of different article formats. In addition, the possibilities of hosting and indexing a journal are discussed. Finally, a user-friendly and future-proof alternative to OJS for supporters of greater openness in scientific publications, PubPub, is presented.

Keywords: open access journal, journal management infrastructure, publication tools

\section{Einführung}

Betrachtet man allein die Entwicklung der ZeitschriftenGründungen bei der beliebtesten Journal-Management und -Publikationssoftware, Open Journal Systems (OJS) ${ }^{1}$, so wird klar, dass nach wie vor zahlreiche neue OpenAccess-Zeitschriften entstehen. Obwohl die Kurve seit dem Jahr 2014 etwas abflacht, gibt es offenbar einen ungebrochenen Trend, alternative, von Verlagen unabhängige wissenschaftliche Zeitschriften auf den Weg zu bringen. ${ }^{2}$ Für publizistische Entrepreneure ist es wichtig, von Anfang an informierte Entscheidungen zu treffen, die den langfristigen Erfolg eines solchen Journals sichern sollen. Besonders bedeutsam werden diese initialen Entscheidungen vor allem technischer Natur dann, wenn es sich um Zeitschriften mit dem Diamond-Open-Access-Geschäftsmodell ${ }^{3}$ handelt, das im Gegensatz zu Gold-OpenAccess darin besteht, dass nicht nur die Leserinnen und Leser sofort bei Erscheinen freien Zugang zu den Artikeln haben, sondern auch die Autoren und Autorinnen kostenlos publizieren können. Oder anders ausgedrückt: Es fallen keine article processing charges (APCs) bzw. submission fees an. ${ }^{4}$ Da die Kasse dieser eher kleinen, im geistes- und sozialwissenschaftlichen Bereich angesiedelten „Indie“-Journals also notorisch leer, die Motivation zur Selbstausbeutung bei den potentiellen Herausgebern und Herausgeberinnen, Redakteurinnen und Redakteuren etc. aber hoch ist, soll hier der Versuch unternommen werden, technische Varianten als Teil eines Leitfadens aufzuzeigen, wie eine Zeitschrift mit minimalem Kosten-

$1 \mathrm{http://pkp.sfu.ca/ojs/.}$

2 https://pkp.sfu.ca/ojs/ojs-usage/ojs-stats/.

3 Fuchs, Christian, Marisol Sandoval: „The Diamond Model of Open Access Publishing: Why Policy Makers, Scholars, Universities, Libraries, Labour Unions and the Publishing World Need to Take Non-Commercial, Non-Profit Open Access Serious.“ In: tripleC 13,2 (2013): 428-443.

4 Gowers, Tim: Discrete Analysis - an arXiv overlay journal. 2015. https://gowers.wordpress.com/2015/09/10/discrete-analysis-an-ar xiv-overlay-journal/. 
aufwand seriös betrieben und maximale Wahrnehmung in der Community erreicht werden kann. Wenngleich dem Diamond-Ansatz nicht immer Erfolg beschieden war viele Journale haben die kritische Phase zwischen dem 6. und 9. Jahrgang nicht überlebt und einige mussten auf APCs, das heißt auf Gold-Open-Access umstellen ${ }^{5}$-, ist er meines Erachtens innovativ und zukunftsträchtig. Neben dem Kostenvorteil bietet er gegenüber Green-Open-Access, wobei es sich häufig um sehr frühe Versionen nicht begutachteter Pre-Prints handelt, auch qualitative Anreize. Diamond-Open-Access-Journals wenden in der Regel ein Editorial- oder Peer-Review-Verfahren an. Zudem haben sie sich dem akademischen Ziel verschrieben, den Zugang zu Wissensproduktion, Wissensverbreitung und Wissensaneignung frei zu gestalten. Die dort Engagierten sollten, da sie sich ehrenamtlich für die unentgeltliche Publikation und Verbreitung von wissenschaftlicher Forschung einsetzen, entsprechenden Nutzen für ihre eigene Karriere daraus ziehen können, Ansehen innerhalb ihrer Community erlangen und Anerkennung von den Institutionen bekommen, in denen sie tätig sind. ${ }^{6}$

Im Bereich der deutschsprachigen Bibliotheks- und Informationswissenschaft wurde in den letzten zehn Jahren eine ganze Reihe von Zeitschriften entweder gleich mit dem Diamond-Geschäftsmodell neu gegründet oder auf dieses Publikationsverfahren umgestellt, z. B. 027.7 Zeitschrift für Bibliothekskultur ${ }^{7}$, Informationspraxis ${ }^{8}$, LIBREAS $^{9}$, o-bib ${ }^{10}$, Perspektive Bibliothek ${ }^{11}$, VÖB Mittei-

5 Björk, Bo-Christer, Cenyu Shen, Mikael Laakso: „A longitudinal study of independent scholar-published open access journals.“ In: PeerJ 4:e1990 (2016). doi:10.7717/peerj.1990.

6 Koroso, Nesru H.: Diamond Open Access. 2015. http://www.uamagazine.com/diamond-open-access/. Ein anderer Ansatz, der Diamond Open Access auch mit höheren Kosten ermöglichen würde, wäre, wenn Forschungsförderer dazu übergehen würden, wissenschaftliche Zeitschriften zu alimentieren, wie sie es mit Forschungsprojekten tun. Vgl. Haspelmath, Martin: How to switch quickly to diamond open access: The best journals are free for authors and readers. 2015. http://www.frank-m-richter.de/freescienceblog/2015/10/28/ how-to-switch-quickly-to-diamond-open-access-the-best-journalsare-free-for-authors-and-readers/. Für weitere alternative Geschäftsmodelle vgl. Smith, Adam: Alternative Open Access Publishing Models: Exploring New Territories in Scholarly Communication. Report on the workshop held on 12 October 2015 at the European Commission Directorate-General for Communications Networks, Content and Technology. 2015. http://wiki.lib.sun.ac.za/images/f/f3/Ec-alternative-oapublishing-models.pdf.

7 http://pubpub.org/0277.

$8 \mathrm{http} / / /$ informationspraxis.de/.

$9 \mathrm{http} / / /$ libreas.eu/.

10 https://www.o-bib.de/.

11 http://journals.ub.uni-heidelberg.de/index.php/bibliothek. lungen ${ }^{12}$ oder kürzlich erst Young Information Scientist ${ }^{13}$. So einmütig das Geschäftsmodell gewählt wurde, so unterschiedlich sind die technischen Umsetzungen. Letzteres gilt über Disziplingrenzen hinaus natürlich umso mehr.

Ein technischer Leitfaden sollte daher folgende, grundlegende Fragestellungen behandeln: Welche Publikationssoftware steht zur Verfügung, welche Tools eignen sich zum Erstellen der Beiträge, wo kann man eine Zeitschrift hosten und wo sollte sie indexiert sein ${ }^{14}$

\section{Software - Journal Management: Alles OJS, oder was?}

Eine der wichtigsten technischen Fragen ist sicherlich, welche (non-proprietäre) Publikationssoftware für eine Open-Access-Zeitschrift verwendet werden soll. Dabei spielt Qualität eine große Rolle, und aus Anwendersicht kann diese anhand der erwarteten „fitness for use“, $d$. h. der subjektiven Gebrauchstauglichkeit gemessen werden. Die ISO Norm 9126 führt als Qualitätsmerkmale Funktionalität, Zuverlässigkeit, Benutzbarkeit, Leistungsfähigkeit, Wartungsfreundlichkeit und Übertragbarkeit auf. ${ }^{15}$ Letztlich wird die Beurteilung immer davon abhängen, ob man ein möglichst einfach zu installierendes System sucht, das intuitiv zu bedienen ist, stabil läuft, wenig Aufmerksamkeit benötigt und viele nützliche Funktionen schon out of the box mitbringt - so dass man praktisch sofort mit dem Publizieren von Heften oder Artikeln beginnen kann, oder ob spezielle Ansprüche an die Ästhetik, das Eingabe- und Publikationsverfahren, den Import bzw. Export von Daten etc. gestellt und so technisch gesehen ein völlig individuelles Journal produziert werden soll - z. B. durch Eingriffe in das CSS oder den Quellcode. Für beide Fälle, und selbstverständlich auch für den Graubereich dazwischen, gibt es Softwarelösungen - sowohl das Rundum-sorglos-Paket wie auch mehrere einzelne Komponenten, die sich kombinieren lassen.

12 https://ojs.univie.ac.at/index.php/voebm/. Diamond Open Access wird auf dieser Seite als „Pure Gold“ bezeichnet.

$13 \mathrm{https} / / /$ yis.univie.ac.at/index.php/yis.

14 Oeinghaus-Steingröver, Hildegard: Aspekte eines Leitfadens zur Entwicklung und Realisierung einer wissenschaftlichen Open-AccessZeitschrift. Diplomarbeit im Studiengang Bibliothekswesen an der Fachhochschule Potsdam. Berlin 2007. $69 \mathrm{ff}$. https://opus4.kobv.de/ opus4-fhpotsdam/frontdoor/deliver/index/docId/53/file/07428.pdf. 15 O’Regan, Gerard: Introduction to Software Quality. Cham 2014. 5-6. 
Neben OJS, das sich zum Standard entwickelt hat, gibt es noch weitere Open-Source-Publikationssoftware, die explizit (auch) für wissenschaftliche Zeitschriften konzipiert wurde und hier in einer Auswahl präsentiert wird. ${ }^{16}$

\subsection{Publikationssysteme speziell für wissen- schaftliche Zeitschriften}

Ambra $^{17}$ ist ein in Java geschriebenes Journal-Management- und Publikationssystem, das von der Public Library of Science (PLOS) aktiv entwickelt wird. Dementsprechend laufen alle Zeitschriften dieser Organisation auf Ambra. Die aktuelle Version 2.11.0 ${ }^{18}$ ist im Mai 2015 erschienen und wartet mit zahlreichen Features auf. So können z. B. von einer Plattform aus mehrere Journals betreut werden, mit dem Journal Publishing Tag Set ${ }^{19}$ der National Library of Medicine (NLM) lassen sich sowohl Inhalt wie auch Metadaten von Artikeln beschreiben, es gibt die Möglichkeit, eine Heftstruktur aufzubauen, Leser- und LeserinnenKommentare abzubilden, Benutzerinnen und Benutzer zu verwalten, Artikel in verschiedenen Zitierstilen zu exportieren und vieles mehr. Wie sich diese Software in der Praxis verhält, lässt sich am besten anhand des wohl bekanntesten Open-Access-Journals überhaupt, PLOS ONE ${ }^{20}$, nachvollziehen. Installation und Konfiguration verlangen allerdings Erfahrung im Umgang mit der Kommandozeile und dürfte daher für Laien eher anspruchsvoll sein.

DPubS (Digital Publishing System) ${ }^{21}$ wurde von der Cornell University Library entwickelt, um Bibliotheken sowie Bibliothekarinnen und Bibliothekaren ein Mittel an die Hand zu geben, den großen, multinationalen, kommerziellen Wissenschaftsverlagen etwas entgegensetzen, selbst als Verlag auftreten und so eine Veränderung im Bereich des wissenschaftlichen Publizierens herbeiführen zu können. ${ }^{22}$ Das Publikationssystem ist modular aufgebaut, gut dokumentiert, enthält verschiedene Services (Editorial, Index, Referral, Registry, User Interface, Admin

16 Auf Repository-Software wie EPrints oder Digital Commons, die sich ebenfalls für das Hosting von Zeitschriften eignet, werde ich im Folgenden nicht eingehen.

17 http://ambraproject.org/.

18 http://ambraproject.org/maven2/release/org/ambraproject/ ambra-webapp/2.11.0/.

19 https://jats.nlm.nih.gov/.

20 http://journals.plos.org/plosone/.

21 http://dpubs.org/.

22 http://dpubs.org/about.html. etc. ${ }^{23}$, ist bereits vorkonfiguriert und kann je nach Bedürfnis angepasst werden. Seit 2009 gibt es allerdings keine neuen Versionen mehr, was die Vermutung nahelegt, dass die Entwicklung eingestellt wurde. Auch für DPubS sind Vorkenntnisse im Aufsetzen von Web-Applikationen hilfreich. ${ }^{24}$ Wie ein Journal mit DPubS präsentiert werden kann, sieht man z. B. auf der Seite des „Project Euclid“25.

Das ePublishing Toolkit $^{26}$ des Living-Reviews-Zeitschriften-Portals der Max-Planck-Gesellschaft ${ }^{27}$ ist ein aus mehreren Komponenten bestehendes Python-Paket, die sich am Lebenszyklus eines Artikels orientieren und unabhängig voneinander genutzt werden können. So stellt es Module für den redaktionellen Workflow (Editorial Management System), die Umwandlung von .tex-Files in PDF und HTML (Publication Builder), bibliografische Daten (Reference Database), Metadaten als RDF-Triples (Register) und die Darstellung im Web bereit (Forms Component, Web Interface, Blogging Component). Heruntergeladen werden kann die Software von zwei Repositorien ${ }^{28}$, Voraussetzungen sind je nach Komponente die Installation von Python, PostgreSQL, Apache, TeX, TeX4ht, bibtool sowie tidy - eine Aufzählung, die technisch weniger versierte Herausgeber und Herausgeberinnen wahrscheinlich abschrecken wird. Wer dennoch einen Blick auf die Software werfen möchte: Die drei Open-Access-Zeitschriften der Living Reviews bieten Gelegenheit dazu. ${ }^{29}$

Lodel (Logiciel d'édition électronique) ${ }^{30}$ hingegen, entwickelt vom Centre pour l'édition électronique ouverte (Cléo), hat den Anspruch, geistes- und sozialwissenschaftlichen Herausgeberinnen und Herausgebern das Erstellen einer Zeitschrift zu ermöglichen, ohne dass sie Computerspezialisten sein müssen. Nach der Installation auf einem Server kann die Software vom Browser aus administriert werden und erlaubt so kollaboratives und - für dezentrale Redaktionsteams - ortsunabhängiges Arbeiten. Die einzelnen Beiträge können lokal mit einem WordProzessor (z. B. LibreOffice, MS Word) oder direkt online erzeugt werden. Lodel ist in der Lage, die mit Textverarbeitungssoftware erstellten Dateien automatisch in XML/ TEI umzuwandeln. Es berücksichtigt zudem die besonde-

23 https://confluence.cornell.edu/display/dpubs/ServiceDescrip tion.

24 https://confluence.cornell.edu/display/dpubs/DPubSInstall Guide.

25 http://projecteuclid.org/.

$26 \mathrm{https}$ ://dev.livingreviews.org/projects/epubtk.

27 Die Living Reviews wurden 2015 vom Verlag Springer gekauft. 28 http://dev.livingreviews.org/repos/epubtk/trunk/ePubTk/ bzw. http://dev.livingreviews.org/repos/epubtk/trunk/ePubTk/webapp/. 29 http://www.livingreviews.org/.

30 https://lodel.org/. 
ren formalen Anforderungen an wissenschaftliche Artikel wie Textstruktur, Fußnoten, Zeichensätze etc. Lodel wird derzeit von 444 wissenschaftlichen Zeitschriften benutzt. Als Systemsprache steht lediglich Französisch zur Verfügung und auch die Dokumentation liegt ausschließlich auf Französisch vor. Das optisch ansprechende Frontend kann im Portal OpenEdition ${ }^{31}$ in Augenschein genommen werden, grafische Einblicke ins Backend liefert ein Aperçu. ${ }^{32}$

\subsection{PHP-Content Management Systeme (CMS)}

Verglichen mit Journal-Management-Systemen, die explizit zum Publizieren wissenschaftlicher Inhalte im Netz erstellt wurden, sind Content-Management-Systeme Alleskönner, die sich an ein breites Publikum mit geringen Programmierkenntnissen zur Veröffentlichung von Inhalten jeglicher Art wenden. ${ }^{33}$ Um ein CMS bildet sich im besten Fall eine (große) Community, welche den Kernbestand an Grundfunktionalitäten weiterentwickelt, regen Austausch pflegt, Tutorials erstellt und das System so für spezifischere Bedarfe einsetzbar macht.

Drupal $^{34}$ ist eines der am weitesten verbreiteten Content-Management-Systeme, das auch zur Publikation wissenschaftlicher Zeitschriften benutzt wird.

Mithilfe der Standardfunktionen und diverser, über einen Browser zusätzlich installierbarer Module kann eine individuelle Publikationsumgebung geschaffen werden. Beginnend bei einer sehr großen Auswahl an freien (responsiven) Weboberflächen (Themes ${ }^{35}$ ), dem anpassbaren Layout (Blockregionen) und dem in über 80 Sprachen verfügbaren Interface, über das Rechte- und Rollenmanagement zur Organisation des Einreichungs- und Review-Prozesses, den HTML-Editor („What you see is what you get“, WYSIWYG), den Export nach EPUB, die Einbindung von Multimedia und die OAI-PMH-Schnittstelle kann Drupal nach den jeweiligen Bedürfnissen konfiguriert und an den Workflow angepasst werden. ${ }^{36}$ Ein Beispiel einer mit Drupal betriebenen, unabhängigen Open-Access-Zeitschrift

$31 \mathrm{http}: / /$ www.revues.org/.

$32 \mathrm{http}: / /$ lodel.org/632.

33 Vgl. eine Liste mit Content Management Systemen unter https:// en.wikipedia.org/wiki/List_of_content_management_systems.

34 https://www.drupal.org/.

35 https://www.drupal.org/project/project_theme.

$36 \mathrm{https} / / /$ open-access.net/fileadmin/oat/oat15/slides/Arning-

Drupal_OA_Tage-Zuerich-Website.pdf. ist Technoculture ${ }^{37}$, aber auch PUBLISSO ${ }^{38}$, das Publikationsportal der ZBMED für die Lebenswissenschaften, verwendet dieses CMS.

Auch mit WordPress ${ }^{39}$, einem weiteren, sehr verbreiteten und flexiblen CMS kann eine wissenschaftliche Zeitschrift betrieben werden, wie man am Beispiel von darkmatter $^{40}$ sieht und wie in einem Blog-Eintrag ${ }^{41}$ dort beschrieben wird. Darüber hinaus existiert mit Annotum ${ }^{42}$ für Wordpress eine eigene Publikationsplattform, welche im CMS fehlende, für das wissenschaftliche Publizieren wichtige Features ergänzt. Annotum, dessen finale Version am 22. November 2016 erschienen ist, hat sich zum Ziel gesetzt, ein einfaches, stabiles und benutzerfreundliches Redaktionssystem für das Erstellen und Veröffentlichen von wissenschaftlichen Artikeln zu entwickeln. Es möchte eine Publikationssoftware zur Verfügung stellen, die es erlaubt, Beiträge einzureichen, $z u$ begutachten und zu publizieren. Insbesondere liefert Annotum u.a. folgende Zusatzfunktionen: einen WYSIWYG-Editor für Formeln, Abbildungen, Tabellen, Zitationen und Literaturnachweise, den Export nach PDF und XML sowie das automatische Erstellen und Registrieren von CrossRef DOIs. ${ }^{43}$

\section{3 ... und dann gibt es da noch OJS}

Von allen Journal-Management-Systemen bringt OJS ${ }^{44}$ offenbar die größte „fitness for use“ mit, hat es sich doch zum unangefochtenen Standard entwickelt. Waren es 1990 weltweit nur 571 Zeitschriften, die mit OJS betrieben wurden, so stieg diese Zahl bis heute auf über $10000 .^{45}$ OJS eignet sich sowohl für Herausgeber und Herausgeberinnen einzelner Journals als auch als Instanz zur Verwaltung mehrerer Zeitschriften im Umfeld von Verlagen oder Institutionen. Die Oberfläche liegt mittlerweile in 35 Sprachen vor, seit der Version 3.0 vom August 2016 sind

37 http://tcjournal.org/drupal/.

$38 \mathrm{http} / / /$ www.publisso.de/.

39 https://wordpress.org/.

$40 \mathrm{http://www.darkmatter101.org/.}$

$41 \mathrm{http} / /$ www.darkmatter101.org/site/2009/12/06/wordpress-asacademic-journal-software/.

$42 \mathrm{https}$ ://annotum.org/. Auch die Integration von OJS als Verwaltungs- in WordPress als Präsentationstool ist möglich. http:// hybridpublishing.org/2014/03/open-journal-system-ojs-integrationin-wordpress-via-plugin/ bzw. https://researchlibrarytechnology. wordpress.com/2014/03/15/open-journal-systems-integration-withwordpress/.

43 https://annotum.wordpress.com/about/.

44 https://pkp.sfu.ca/ojs/.

45 https://pkp.sfu.ca/ojs/ojs-usage/ojs-stats/. 
Basis-Themes im Responsive Design (Bootstrap) enthalten ${ }^{46}$, und das Installieren der Software bzw. Einrichten eines Journals im Browser ist vergleichsweise einfach. ${ }^{47}$ Vom Einreichen eines Manuskripts über die Begutachtung (Editorial Review, Peer-Review, Open (Peer) Review) bis hin zur Publikation können komplexe Abläufe angelegt und vielfältige Rollen (Zeitschriftenverwaltung, Redaktion, Rubrikredaktion, Gutachten, Autor/Autorin, Leserin/ Leser, Abonnementverwaltung) vergeben werden. Außerdem enthält OJS zahlreiche Plug-ins für den Daten-Export und -Import (z.B. XML, DOAJ, DataCite, CrossRef). Im deutschsprachigen Raum hat sich das DFG-Projekt „OJSde.net“ zur Aufgabe gemacht, die Nutzung dieses Publikationssystems durch diverse Services ${ }^{48}$ weiter zu erleichtern. Einen Überblick über Aussehen und Handhabung von OJS kann man sich auf der Website des Public Knowledge Projects verschaffen. ${ }^{49}$

\section{Software-Tools}

Je nachdem, in welchen und in wie vielen Dateiformaten die Artikel einer Zeitschrift erscheinen sollen, braucht man bestimmte Tools zum Erstellen bzw. Konvertieren der Files. Exemplarisch werden hier Werkzeuge für drei gängige Formate vorgestellt.

\subsection{PDF-Version erstellen}

PDF ist ein häufig gewähltes Publikationsformat, da es mit Hilfe von Word-Prozessoren (z. B. LibreOffice, Google Docs) leicht zu erstellen ist. Statt mit WYSIWYG-Editoren können PDFs aber auch mit dem Textsatzsystem LaTeX kreiert werden, das auf dem Prinzip ,What you see is what you mean" (WYSIWYM) beruht. Während herkömmliche Textverarbeitungssoftware auf den universellen Einsatz für ein Massenpublikum zugeschnitten ist, wurde LaTeX speziell für wissenschaftliches Schreiben konzipiert. Es liefert sehr gut strukturierte Dokumente von hoher typografischer Qualität. Um LaTeX zu benutzen, benötigt man

46 Für die Vorgängerversionen 2.4.x hat die George Mason University ein frei verfügbares Responsive Theme entworfen. https://github. com/masonpublishing/OJS-Theme.

47 https://pkp.sfu.ca/ojs/docs/userguide/2.3.1/systemAdministra tionInstallProcess.html.

$48 \mathrm{http}: / /$ www.ojs-de.net/services/index.html.

49 https://pkp.sfu.ca/ojs/ojs_demo/. das TeX-Grundsystem (TeX Live ${ }^{50}$, MacTeX ${ }^{51}$ bzw. Mik$\mathrm{TeX}^{52}$ ) und einen LaTeX-Editor, z. B. den plattformunabhängigen Texmaker ${ }^{53}$. Webbasierte Software wie Overleaf oder ShareLaTeX erspart die Installation des TeX-Grundsystems, bietet durch Live-Kompilierung auch WYSIWYGFunktionalität und ermöglicht das kollaborative Editieren von Texten. Die Entscheidung, mit welchem System eine Zeitschrift ihre Dokumente zur Publikation vorbereitet, sollte jedoch gut überlegt sein: LaTeX kann sich verglichen mit herkömmlichen Word-Prozessoren als zeitraubende Angelegenheit erweisen. ${ }^{54}$

\subsection{HTML-Version erstellen}

Bestenfalls in Verbindung mit einer für mobile Geräte optimierten Benutzeroberfläche des Journal-ManagementSystems bietet sich HTML als ein klassisches Publikationsformat an. Benötigt wird lediglich ein HTML-Editor, in den man entweder direkt Quellcode (WYSIWYM) oder Text (WYSIWYG) eingeben kann. ${ }^{55}$ Die Darstellung erfolgt dann über den Web-Browser. Als Nachteil für wissenschaftliche Publikationen erweist sich z. B., dass Fußnoten in HTML als Hyperlinks angelegt werden müssen und Literaturverzeichnisse nicht automatisch generiert werden können. Vorteilhaft sind die unkomplizierte Einbindung von Multimedia-Inhalten sowie die einfache Durchsuch- und Verlinkbarkeit von Artikeln.

\subsection{EPUB-Version erstellen}

Falls die Beiträge eines Journals zusätzlich noch in einem für E-Book-Reader und andere mobile Endgeräte interpretierbaren, dynamischen Format erscheinen und gewissermaßen die Vorteile von PDF und HTML kombinieren sollen, bietet sich EPUB als offener Standard an. Das E-Book-Management-Tool Calibre ${ }^{56}$ ist z. B. in der Lage, PDFs oder HTMLs in EPUBs, MOBIs und viele weitere For-

50 https://www.tug.org/texlive/.

51 http://www.tug.org/mactex/.

52 https://miktex.org/.

53 http://www.xm1math.net/texmaker/ (19.12.2016). Vgl. eine Liste mit LaTeX-Editoren und deren technischen Features unter: https:// en.wikipedia.org/wiki/Comparison_of_TeX_editors.

54 Knauff, Markus, Jelica Nejasmic: „An Efficiency Comparison of Document Preparation Systems Used in Academic Research and Development.“ In: PLOS ONE 10,4 (2014). doi:10.1371/journal. pone.0125830.

55 Vgl. eine Liste von HTML-Editoren: https://de.wikipedia.org/ wiki/Liste_von_HTML-Editoren.

56 https://calibre-ebook.com/. 
mate umzuwandeln. Für die Nachbearbeitung kann ein eingebauter Editor benutzt werden.

\subsection{Formate automatisch konvertieren}

Schön wäre es, wenn man mit Hilfe eines einzigen Tools einen Beitrag aus einem Basis-Format automatisch und verlustfrei in weitere Formate konvertieren könnte, also z.B. von LaTeX (.tex, PDF) nach HTML und EPUB. Gerade dieser Umwandlungsprozess entpuppt sich nämlich häufig als zeitraubend, frustrierend und - sofern manuell bewerkstelligt - fehleranfällig. Mit Pandoc ${ }^{57}$ steht ein Tool zur Verfügung, das z. B. aus HTML-, LaTeX-, docx- oder odt-Dokumenten EPUBs, XML-Dateien, Markdown oder PDFs u.v. m. machen kann und umgekehrt. Zusätzlich „versteht“ es syntaktische Erweiterungen (Markdown) für Metadaten (Titel, Autor, Datum), Fußnoten, Tabellen, Listen, Exponenten, tiefgestellte Zeichen etc. und enthält ein System für automatisches Zitieren in verschiedenen Stilen und das Erstellen des Literaturverzeichnisses. Das Programm hat kein grafisches User Interface und muss in der Kommandozeile ausgeführt werden. Zum Ausprobieren wird aber ein Online-Konverter angeboten. ${ }^{58}$

\section{Server - Wo hosten?}

Das Charakteristische der bisher vorgestellten Journal-Management-Software bzw. der Content-Management-Systeme ist, dass sie heruntergeladen und lokal installiert und betrieben werden müssen. Es braucht daher einen Server, um sie zu hosten.

Einerseits gibt es dafür kommerzielle Lösungen, etwa das Mieten von Speicherplatz bei Hosting-Providern, zum Teil schon für kleines Geld. Wer Installation und Administration des Systems selbst in die Hand nimmt, profitiert vom Unterbietungswettkampf der verschiedenen Anbieter und bekommt die Wunsch-URL gleich mitgeliefert. Allerdings sollte er oder sie dann auch in der Lage sein, Patches einzuspielen bzw. Versions-Updates vorzunehmen, um Sicherheitslücken zu schließen. Speziell im CMS-Bereich besteht die Möglichkeit, die bevorzugte Software gleich vom Provider installieren und pflegen zu lassen, um sich voll und ganz auf das Layout und den Inhalt der Zeitschrift konzentrieren zu können. Neuerdings

$57 \mathrm{http}: / /$ pandoc.org/. $58 \mathrm{http}: / /$ pandoc.org/try/. bietet das Public Knowledge Project (PKP) kostenpflichtige Hosting-Services für OJS-Journals auf Servern der Simon-Frazer-Universität bzw. eines anderen Dienstleisters in Kanada an. Die günstigste Variante liegt bei 850 Kanadischen Dollar jährlich pro Zeitschrift und umfasst eine OJS-Installation, 5 GB Speicherplatz, Upgrades, Sicherheitsaktualisierungen, tägliche Backups und die für die Langzeitarchivierung der Artikel so wichtige Mitgliedschaft im privaten LOCKSS-Netzwerk (PLN) von PKP. Letztere steht seit der OJS-Version 2.4.8 über ein Plug-in aber auch jenen unabhängigen OJS-Journals gebührenfrei offen, die mindestens einen Artikel publiziert haben und über eine ISSN verfügen (die man von der Deutschen Nationalbibliothek ${ }^{59}$, der Schweizerischen Nationalbibliothek ${ }^{60}$ bzw. dem ISSN International Centre ${ }^{61}$ in Paris bekommt).

Daneben existieren - das in Kapitel 6 noch vorzustellende Tool nicht mitgerechnet - aber auch zwei kostenlose Alternativen, eine Zeitschrift zu hosten. Zum einen nennen inzwischen über 80 Bildungseinrichtungen im deutschsprachigen Raum ${ }^{62}$, vornehmlich wissenschaftliche Bibliotheken, OJS-Installationen ihr Eigen und sind offenbar bereit, auch „externe“ Publikationen mit zu betreuen - wie z.B. im Fall der „Informationspraxis“, die von der Universitätsbibliothek Heidelberg gehostet wird, obwohl kein Redaktionsmitglied eine institutionelle Anbindung hat. ${ }^{63}$ Eine Anfrage an entsprechende OJS-Standorte könnte also durchaus erfolgversprechend sein. Zum anderen eignet sich auch GitHub, wenngleich ein wenig zweckentfremdet, weil ursprünglich für Software-Entwicklungsprojekte gedacht, für das JournalHosting, wie u.a. LIBREAS, das ReScience Journal ${ }^{64}$ und das Journal of Open Source Software ${ }^{65}$ eindrücklich beweisen.

\section{Suchmaschine - Indexierung}

Für die möglichst große Verbreitung der Inhalte einer Zeitschrift ist es erforderlich, in wissenschaftlichen Suchmaschinen, bibliografischen Fachdatenbanken und Repositorien, d.h. in Abstracting und Indexing (A\&I) Services

$59 \mathrm{http} / / /$ www.dnb.de/DE/Wir/Kooperation/ISSN/issn_node.html. $60 \mathrm{http}: / /$ www.nb.admin.ch/nb_professionnel/issn/.

$61 \mathrm{http}: / /$ www.issn.org/.

$62 \mathrm{http} / / /$ www.ojs-de.net/netzwerk/Standorte/index.html.

63 https://journals.ub.uni-heidelberg.de/index.php/ip/about/edi torialTeam.

$64 \mathrm{http}: / /$ rescience.github.io/.

65 http://joss.theoj.org/. 
präsent zu sein. ${ }^{66}$ Die beiden wichtigsten Vertreter dieser Gattungen im unabhängigen Open-Access-Bereich dürften wohl Google Scholar und das Directory of Open Access Journals (DOAJ) sein.

Grundsätzlich sind Google Scholar Publikationen aus verschiedensten Quellen, sämtlichen Fachgebieten und Ländern, in allen Sprachen und aus allen Epochen willkommen, solange sie zwei Kriterien erfüllen: erstens muss es sich um wissenschaftliche Beiträge, also Zeitschriftenartikel, Tagungsbeiträge, technische Berichte u. ä. handeln; zweitens muss entweder der ganze Artikel als Volltext (Google versteht darunter PDF- oder HTMLVersionen) oder mindestens dessen Abstract frei zugänglich und prominent platziert sein. Die Dateigröße darf nicht mehr als $5 \mathrm{MB}$ betragen. ${ }^{67}$ Technisch versierten Herausgeberinnen und Herausgebern, die ihr Journal nicht in einem Open-Access-Verlag, sondern auf ihrer eigenen Webseite betreiben möchten, wird von Google Scholar ausdrücklich OJS als Journal-Management-System empfohlen. ${ }^{68}$

DOAJ enthält derzeit über 9000 Journals und fast 2,5 Millionen Artikel. ${ }^{69} \mathrm{Um}$ die Aufnahme einer Zeitschrift muss man sich durch das sorgfältige Ausfüllen eines Formulars bewerben. ${ }^{70}$ Dort werden mehr als 50 Parameter abgefragt, die Entscheidungsgrundlage dafür sind, ob ein Journal akzeptiert oder abgelehnt wird. Besonders „Vorbildliche“ Periodika erhalten ein spezielles Gütesiegel, das sich auf formale Attribute wie $\mathrm{Zu}-$ gang, Offenheit, Auffindbarkeit, Wiederverwendbarkeit und Autorenrechte bezieht - aber ausdrücklich nicht auf die inhaltliche Qualität der publizierten Artikel. Unabdingbare Voraussetzungen für einen Eintrag in DOAJ sind $u$. a. eine ISSN, eine URL pro Zeitschrift, eine eindeutige URL pro Artikel sowie eine HTML- und/oder PDF-Version pro Artikel. ${ }^{71}$ Die Herausgeberinnen und Herausgeber sind nach erfolgter Registrierung dafür verantwortlich, die Metadaten der Beiträge über eine Einga-

66 Gaetz, Ivan: „Processes, Opportunities, and Challenges Creating and Managing a Scholarly Open Access Journal: An Investigation of ,Collaborative Librarianship،“" In: Space and Organizational Considerations in Academic Library Partnerships and Collaborations. Hrsg. von Brian Doherty. 205-229, hier 220. Hershey PA 2016. 67 https://scholar.google.com/intl/en/scholar/inclusion. html\#content.

68 https://scholar.google.com/intl/en/scholar/inclusion. html\#overview.

69 https://doaj.org/.

$70 \mathrm{https} / / /$ doaj.org/application/new.

71 Für weitere Voraussetzungen vgl. https://doaj.org/publishers. bemaske in DOAJ einzutragen oder sie über ein XML-File hochzuladen. ${ }^{72}$

Über DOAJ (oder die OAI-Schnittstelle des JournalPublication-Systems) gelangen die Inhalte außerdem in weitere wissenschaftliche Suchmaschinen wie die Bielefeld Academic Search Engine (BASE) ${ }^{73}$ oder Microsoft Academic $^{74}$, in Web-Scale Discovery Services wie z. B. den Summon-Index ${ }^{75}$ und dadurch wiederum in Bibliothekskataloge. Durch Lizenzverträge mit führenden Informationsanbietern können sie auch Eingang in subskriptionspflichtige bibliografische Fachdatenbanken finden. ${ }^{76}$ Grundsätzlich gilt: an je mehr renommierten Orten eine Zeitschrift gelistet ist, desto mehr Aufmerksamkeit werden die Inhalte bekommen und desto einfacher wird es sein, Autorinnen und Autoren für Beiträge zu gewinnen.

Durch die Vergabe eines Digital Object Identifier (DOI) für Artikel wird deren Verfügbarkeit auch dann sichergestellt, wenn sich die Domain einer Zeitschrift ändert oder sie ganz eingestellt wird. Bezogen werden können DOIs von Registrierungsagenturen wie z. B. DataCite oder Crossref - die auch für die Indexierung, das Exportieren und die Durchsuchbarkeit der Metadaten sorgen - allerdings braucht es hierfür eine institutionelle Anbindung, da Einzelpersonen keine DOIs zugeteilt werden. ${ }^{77}$

\section{PubPub als , radikale“ Alterna- tive zu OJS?}

Seit 2016 wird am Media Lab des Massachusetts Institute of Technology (MIT) die Publikationssoftware PubPub ${ }^{78}$ entwickelt. Das Projekt geht von der provokanten These aus, dass das bisherige akademische Publikationsgebaren zu Ineffizienz (inefficiency) und Verfälschung (corruption) geführt, und so dem Streben nach wissenschaftlicher Erkenntnis geschadet (detriment) hat. ${ }^{79}$ Zeitschriften sollen künftig offene Inhalte kuratieren, statt sich als Wäch-

72 Cheby, Lisa E.: „Open Access Metadata for Journals in Directory of Open Access Journals: Who, How, and What Scheme?" In: SLIS Student Research Journal 6,1 (2016). http://scholarworks.sjsu.edu/ slissrj/vol6/iss1/4. OJS verfügt bereits über ein DOAJ-Export-Plug-In. 73 https://www.base-search.net/.

74 https://academic.microsoft.com/.

$75 \mathrm{http}$ ://www.proquest.com/products-services/The-SummonService.html.

76 Die Inhalte aus 027.7 sind bspw. in EBSCO's Library \& Information Science Source indexiert. https://www.ebscohost.com/titleLists/llscoverage.pdf.

77 http://search.datacite.org/ und http://www.crossref.org/.

78 https://www.pubpub.org/.

79 https://www.pubpub.org/pub/tos. 
ter über den wissenschaftlichen Fortschritt zu begreifen. Dieser „demokratische“ Ansatz hat zur Folge, dass nicht mehr nur Institutionen, sondern auch Individuen in die Lage versetzt werden, unkompliziert Journals für bestimmte Interessengruppen $\mathrm{zu}$ gründen (grassroots journals). Die Rechte an den Artikeln (genannt „Pubs“) bleiben uneingeschränkt bei den Autoren und Autorinnen, aber die Beiträge können von Herausgeberinnen und Herausgebern in beliebig viele mit PubPub erstellte Zeitschriften aufgenommen werden. Das Tool ermöglicht das gemeinschaftliche Bearbeiten von Dokumenten (collaborative editing), schnelles Publizieren (instant publishing) sowie die kontinuierliche Überprüfung (continuous review) der Inhalte.

In der technischen Umsetzung manifestieren sich diese Voraussetzungen durch markante Unterschiede zu den vorher beschriebenen Journal-Management-Systemen. PubPub wird als kostenlose „Software as a Service“ (SaaS) angeboten, muss also nicht lokal installiert werden. Der Zugang zur Plattform erfolgt denkbar einfach über das Anlegen eines Accounts, und auch eine Zeitschrift ist nach Angabe des Titels, der URL (pubpub.org/...) und ggf. einer kurzen Beschreibung schnell eingerichtet. ${ }^{80}$ Über einen WYSIWYG-Editor können Pubs verfasst, publiziert und verwaltet werden. Er erlaubt es auch, Dokumente, Tabellen, Literaturnachweise, Multimedia (Abbildungen, Videos) und Code (Iframe, Jupyter, LaTeX) über den Browser hochzuladen bzw. einzubinden. In einem Panel neben dem Artikel wird dessen Struktur zur Navigation abgebildet und es gestattet, ihn anhand von Kommentaren oder offenen Peer-Reviews, die z. B. von den Herausgebern und Herausgeberinnen gezielt bei Gutachterinnen und Gutachtern angefordert werden, zu diskutieren. Sollten daraufhin Änderungen am Beitrag vorgenommen worden sein, sind diese aufgrund der vorhandenen Versionierung nachvollziehbar, d.h. der gesamte Entwicklungsprozess eines Pubs ist dokumentiert. Als Rollen können im Backend Beitragende, Leser oder Leserin, Herausgeberin oder Herausgeber und Autor oder Autorin zugewiesen werden. Pubs lassen sich automatisch nach PDF und Markdown exportieren, als Zitierstile stehen neben BibTeX auch APA, MLA und Chicago Style zur Verfügung. Man kann sowohl einem Journal wie auch einzelnen Pubs folgen. Bei der Veröffentlichung eines Artikels wird für diese Version ein DOI vergeben. Daten und Inhalte der Zeitschriften liegen auf Servern des MIT, können aber mittels einer API zur Gestaltung einer völlig eigenständigen Journal-Homepage verwendet werden.

80 https://www.pubpub.org/journals/create.
Zum jetzigen Zeitpunkt ist PubPub für wissenschaftliche Zeitschriften nur bedingt einsetzbar, da viele der angesprochenen Features noch in Entwicklung begriffen sind und nicht zufriedenstellend funktionieren. Sollte sich das wissenschaftliche Publikationswesen jedoch weiter in Richtung von mehr Offenheit und Transparenz verändern, könnte sich PubPub zu einem ernsthaften Konkurrenten von OJS entwickeln, das eher dem traditionellen Publikationsprozess verhaftet und verglichen mit PubPub sperriger zu bedienen ist.

\section{Fazit}

Von der Idee bis zur erfolgreichen Umsetzung eines OpenAccess-Journals gilt es im Vorfeld viele Faktoren zu berücksichtigen. In einem Business-Plan, wo Überlegungen zu den erforderlichen Ressourcen, dem „Marktumfeld“, den „Eigentumsverhältnissen“ und Zuständigkeiten, dem Marketing, der Finanzierung und den Risiken einer solchen Unternehmung anzustellen sind, sind zwingend auch technische Fragen zu beantworten. So beeinflussen etwa die Entscheidung für ein bestimmtes Peer-ReviewVerfahren, die Einschätzung der eigenen IT-Kompetenz und der Wunsch nach Verbreitung bzw. Auffindbarkeit der Inhalte die Wahl der geeigneten Publikationsumgebung wesentlich. ${ }^{81}$

Ziel der vorliegenden Zusammenstellung war es, diese Wahl zu erleichtern und mögliche Hürden oder Fallstricke von vornherein zu umgehen. Des Weiteren sollte aufgezeigt werden, dass eine Fülle an non-proprietären Tools vorhanden ist, die es ermöglicht, die für die eigenen Bedürfnisse optimale Publikationsinfrastruktur aufzubauen bzw. zu nutzen.

Wenn man allgemein die erforderlichen individuellen technischen Fähigkeiten zu den jährlichen Kosten in Beziehung setzt, dann lässt sich das Verhältnis für ein völlig individuell eingerichtetes Journal-ManagementSystem grob als mittel/niedrig-mittel, für ein integriertes System wie OJS als niedrig/hoch und für ein mit anderen Tools kombiniertes, in Teilen integriertes System als mittel-hoch/mittel-hoch kategorisieren. ${ }^{82}$ Neuerdings existiert mit PubPub aber auch eine Lösung, die weder viel

81 Brown, Sheridan: Business Plan Toolkit. Publishing an Open Access Journal. Prepared for SPARC Europe. 2016. http://sparceurope. org/wp-content/uploads/2016/01/BusinessPLAN_OAJournals_0116. pdf.

82 Hybrid Publishing Lab; Centre for Digital Cultures, Leuphana University of Lüneburg: How to Start an Open Access Journal. http:// hybridpublishing.org/files/2014/07/HOAJ-POSTER-final-web.pdf. 
technisches Know-how voraussetzt noch Hosting-Kosten verursacht (niedrig/niedrig). Und was schließlich die Außenwirkung betrifft, dürfte eine neue, z. B. mit PubPub publizierte Zeitschrift deutlich mehr Aufsehen erregen als das x-te OJS-Journal.

\section{Autoreninformationen}

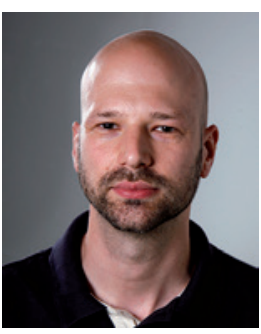

\section{Dr. Andreas Ledl}

Universitätsbibliothek Basel

Schönbeinstraße 18-20

4056 Basel

Schweiz

andreas.ledl@unibas.ch

orcid.org/0000-0002-0629-0446 